\title{
Structural Correlates of Efficient GABAergic Transmission in the Basal Ganglia-Thalamus Pathway
}

\author{
Ágnes L. Bodor, ${ }^{1}$ Kristóf Giber, ${ }^{1}$ Zita Rovó, ${ }^{1}$ István Ulbert, ${ }^{2,3}$ and László Acsády ${ }^{1}$ \\ ${ }^{1}$ Department of Cellular and Network Neurobiology, Institute of Experimental Medicine, Hungarian Academy of Sciences, H-1450 Budapest, Hungary, \\ 2Institute of Psychology, Hungarian Academy of Sciences, 1068 Budapest, Hungary, and 32Department of Information Technology, Péter Pázmány Catholic \\ University, H-1083 Budapest, Hungary
}

Giant inhibitory terminals with multiple synapses, the counterparts of excitatory "detonator" or "driver" terminals, have not been described in the forebrain. Using three-dimensional reconstructions of electron microscopic images, we quantitatively characterize a GABAergic pathway that establishes synaptic contacts exclusively via multiple synapses. Axon terminals of the nigrothalamic pathway formed, on average, 8.5 synapses on large-diameter dendrites and somata of relay cells in the ventromedial nucleus of the rat thalamus. All synapses of a given terminal converged on a single postsynaptic element. The vast majority of the synapses established by a single terminal were not separated by astrocytic processes. Nigrothalamic terminals in the macaque monkey showed the same ultrastructural features both in qualitative and quantitative terms (the median number of synapse per target was also 8.5). The individual synapses were closely spaced in both species. The nearest-neighbor synaptic distances were $169 \mathrm{~nm}$ in the rat and $178 \mathrm{~nm}$ in the monkey. The average number of synapses within $0.75 \mu \mathrm{m}$ from any given synapse was 3.8 in the rat and 3.5 in the monkey. The arrangement of synapses described in this study creates favorable conditions for intersynaptic spillover of GABA among the multiple synapses of a single bouton, which can result in larger charge transfer. This could explain faithful and efficient GABAergic signal transmission in the nigrothalamic pathway in the healthy condition and during Parkinson's disease. In addition, our structural data suggest that the rodent nigrothalamic pathway can be a valid model of the primate condition, when the mechanism of GABAergic transmission is studied.

Key words: spillover; driver; axon terminal; active zone; substantia nigra; Parkinson's disease

\section{Introduction}

Postsynaptic responses critically depend on the type of presynaptic terminals and the number and arrangement of synapses (Cathala et al., 2005; for review, see Rollenhagen and Lübke, 2006). In case of excitatory transmission, these parameters show great variability. Most glutamatergic terminals are small and establish contacts via single or few synapses on their postsynaptic targets (Shepherd and Harris, 1998). However, giant excitatory terminals, which target proximal dendritic regions or somata and establish contacts via a large number of synapses, have also been described (Chicurel and Harris, 1992; Satzler et al., 2002; Cathala et al., 2005). These "detonator" or "driver" terminals of the brainstem, cerebellum, thalamus, or cortex have a large impact on their postsynaptic targets (Sherman and Guillery, 1998). This enables faithful synaptic transmission and frequently a one-to-one spike

\footnotetext{
Received Aug. 29, 2007; revised Feb. 2, 2008; accepted Feb. 4, 2008.

This work was supported by the Wellcome Trust (L.A. is in receipt of a Wellcome Trust International Senior Research Fellowship), the Hungarian Scientific Research Fund (OTKA 49100, 64184), Institute du Cerveau et de la Moelle épiniere, and the National Office for Research and Technology (Program Öveges), Neuroprobes FP6. We are grateful to Dr. Balázs Hangya for help in the statistical analysis and to Krisztina Faddi and Gyöző Goda for excellent technical assistance. We thank Profs. David Perkel, Zoltán Nusser, and Anita Lüthi, Dr. Hajnalka Bokor, and Andrea Slézia for comments on this manuscript.

This article is freely available online through the J Neurosci Open Choice option.

Correspondence should be addressed to Dr. László Acsády, Institute of Experimental Medicine, Hungarian Academy of Sciences, Szigony u. 43, H-1083, Budapest, Hungary. E-mail: acsady@koki.hu.

D0I:10.1523/JNEUROSC1.5266-07.2008

Copyright $\odot 2008$ Society for Neuroscience $\quad 0270-6474 / 08 / 283090-13 \$ 15.00 / 0$
}

coupling between the presynaptic and postsynaptic action potentials (Smith et al., 1998; Henze et al., 2002; Deschenes et al., 2003).

The heterogeneity of GABAergic terminals is much less explored. The available three-dimensional (3D) data suggest that most GABAergic terminals establish contacts via single or few synapses (Kubota and Kawaguchi, 2000; Biro et al., 2006). A multisite GABAergic terminal type has been described in the cerebellum (Telgkamp et al., 2004), but giant GABAergic terminals comparable to their excitatory counterpart have not been quantitatively characterized in the forebrain. Combined anatomical and physiological data demonstrated that large, presumably multisite GABAergic terminals are present in the thalamus and exert strong inhibitory influence on their targets (Bartho et al., 2002; Bokor et al., 2005; Lavallee et al., 2005). Analysis of single electron microscopic sections indicated that certain thalamic boutons of nigral origin may also establish multiple synapses (Kultas-Ilinsky and Ilinsky, 1990; Kuroda and Price, 1991; Sakai et al., 1998; Tsumori et al., 2002), but quantitative characterization of the number and distribution of these synapses is missing.

The nigrothalamic pathway represents one of the major outputs of the basal ganglia (Di Chiara et al., 1979; Tepper et al., 2007). It transmits a highly condensed code of the original cortical output signal to the motor thalamus, which is the final relay station of the cortex-basal ganglia-thalamus-cortex loop (BarGad et al., 2003). The nigrothalamic pathway exerts strong inhibitory control on thalamocortical cells (Chevalier and Deniau, 
1982; Ueki, 1983), which have a large impact on cortical activity (Paz et al., 2007). The structural basis of the effectiveness of the nigrothalamic pathway, however, has not been elucidated yet. Single-section analysis does not provide information about the number and arrangement of synapses and the morphological heterogeneity of the terminals, all of which are indispensable to understand transmitter dynamics and postsynaptic responses (for review, see Rollenhagen and Lübke, 2006). Indeed, the kinetics of the postsynaptic response primarily depends on the size (Taschenberger et al., 2002) and exact position of synapses relative to each other (Cathala et al., 2005). Thus, in the present study, we performed a 3D electron microscopic study of nigrothalamic terminals, which allowed detailed quantitative analysis of the number and arrangement of synapses. Our data disclosed a large, peculiar GABAergic terminal type previously unknown in the mammalian forebrain.

\section{Materials and Methods}

All experimental procedures on rats were performed according to the ethical guidelines of the Institute of Experimental Medicine, Hungarian Academy of Sciences and approved by the Ethical Committee. All primate experiments, surgical rooms, and housing conditions were approved by the local ethical committee of the Institute of Psychology, Hungarian Academy of Sciences and by the Animal Health and Food Control Station, Budapest.

Surgery and the perfusion of rats. Adult, male Wistar rats (250-300 g; $n=7$ ) were deeply anesthetized with Equithesin (in \%: 4.2 chloral hydrate, $2.12 \mathrm{MgSO}_{4}, 16.2 \mathrm{Nembutal}, 39.6$ propylene glycol, and 10 ethanol in $\mathrm{H}_{2} \mathrm{O} ; 0.3 \mathrm{ml} / 100$ g, i.p.) and placed in a stereotaxic frame. Iontophoretic injections of anterograde tracers (15-25 min, 0.5-4.0 $\mu \mathrm{A}, 2-7 \mathrm{~s}$ on/off duty cycle) were made via a glass capillary (tip outer diameter, $15-50 \mu \mathrm{m}$ ) into the substantia nigra pars reticulata (SNR) at the following coordinates relative to bregma 0 point according to the atlas of Paxinos and Watson (1998): posterior, -4.7 to $-5.4 \mathrm{~mm}$; lateral, $2.2-2.4$ $\mathrm{mm}$; ventral, -7.7 to $-8.2 \mathrm{~mm}$. Four unilateral and three bilateral injections were performed. Two different anterograde tracers, biotinylated dextran amine [BDA; 10,000 molecular weight; $10 \%$ in $0.1 \mathrm{M}$ phosphate buffer (PB), pH 7.4; $n=9$; Invitrogen, Leiden, The Netherlands] and Phaseolus vulgaris leucoagglutinin (PHAL; $2.5 \%$ in $0.01 \mathrm{M} \mathrm{PB;} n=1$; Vector Laboratories, Burlingame, CA), were used to label the nigrothalamic pathway. After a survival time of 5-7 d, rats were deeply anesthetized with Equithesin and perfused through the heart, first with physiological saline ( $3 \mathrm{~min}$ ), followed by $100 \mathrm{ml}$ of fixative containing $2 \%$ paraformaldehyde (TAAB, Berks, UK) and $0.5 \%$ glutaraldehyde (TAAB) in acetate buffer ( $\mathrm{pH} 6.0 ; 5 \mathrm{~min}$ ), and finally with $500 \mathrm{ml}$ of fixative containing $2 \%$ paraformaldehyde and $0.5 \%$ glutaraldehyde in borate buffer (pH 8.5; 50 min) (Berod et al., 1981).

Preembedding immunocytochemistry for light microscopy. After perfusion, brains were removed from the skull, and coronal sections (50-60 $\mu \mathrm{m}$ thick) containing the thalamus and the SNR were cut with a vibratome. After washes in PB (five times for $10 \mathrm{~min}$ ), sections were incubated in sucrose (30\%) overnight, followed by freeze-thawing over liquid nitrogen. After additional washes in PB (three times for $10 \mathrm{~min}$ ), the sections were treated with sodium borohydride $(0.5 \% ; 15$ min in $\mathrm{PB})$. The rest of the washes and dilutions of antisera were done in $0.05 \mathrm{M}$ Tris-buffered saline (TBS), $\mathrm{pH}$ 7.4.

Injection sites and labeled fibers were visualized with a nickelintensified 3,3'-diaminobenzidine (DABNi) reaction resulting in a bluish-black reaction product. In the case of BDA-injected animals $(n=$ 6), sections were incubated with avidin biotinylated-horseradish peroxidase complex (ABC; 1:300; Vector Laboratories) for $2 \mathrm{~h}$ and developed with DABNi. Sections from animals injected by PHAL $(n=1)$ were first incubated with rabbit anti-PHAL (1:10,000; Vector Laboratories) overnight, followed by biotinylated goat anti-rabbit (1:300; Vector Laboratories), $\mathrm{ABC}$, and $\mathrm{DABNi}$. The affinity-purified rabbit anti-PHAL antiserum (code number AS 2300; Vector Laboratories) was prepared against the plant protein Phaseolus vulgaris agglutinin, which is normally not present in the brain. Brain regions free of the tracer showed absolutely no immunostaining, demonstrating the specificity of the antibody.

Injection sites of the anterograde tracers (nine BDA and one PHAL; diameter, $300-600 \mu \mathrm{m}$ ) were localized in the SNR in 10 cases and were placed at several levels along the anteroposterior extent of the SNR (see Fig. $1 \mathrm{~A}$ ). The small unilateral injections resulted in hardly any contralateral fiber labeling. In one case, labeling of cell bodies and terminals was observed in the subthalamic nucleus. Because SNR does not provide strong input to the subthalamic nucleus, these terminals represent the local collaterals of retrogradely labeled subthalamic neurons as a result of spurious collateral labeling.

Double labeling was performed to map the codistribution of calbindin and the labeled terminals in the thalamus. After visualizing the tracer with $\mathrm{DABNi}$, sections containing anterogradely labeled terminals were incubated with mouse anti-calbindin antiserum (1:4000, overnight; Swant, Bellinzona, Switzerland). Next, the sections were treated with biotinylated anti-mouse IgG made in goat (1:300, 2 h; Vector Laboratories) and then with $\mathrm{ABC}$. Finally, the immunoperoxidase reaction was developed using $\mathrm{DAB}$ as the chromogen, which gave a brown reaction product. The mouse anti-calbindin antibody (code number 300; Swant) was prepared by the hybridization of mouse myeloma cells with spleen cells from mice immunized with calbindin D-28k purified from chicken gut. This calcium-binding protein antibody showed identical staining to previous descriptions obtained by rabbit antisera raised against different parts of the protein (Baimbridge and Miller, 1982).

Sections were then treated with $\mathrm{OsO}_{4}(1 \%$ in $0.1 \mathrm{~m} \mathrm{~PB} ; 45 \mathrm{~min})$ containing $7 \%$ glucose, dehydrated in ethanol and propylene oxide, and embedded in Durcupan (ACM; Fluka, Buchs, Switzerland). Glucose treatment allowed the preservance of the color differences between black (DABNi) and brown (DAB) precipitates (see Fig. $1 I$ ).

Light microscopic images were taken with a digital camera [Olympus (Tokyo, Japan) DP 70 or Zeiss (Budapest, Hungary) AxioCam HRc]. Brightness and contrast were adjusted when necessary using Adobe (San Jose, CA) Photoshop 7.0, applied to entire images only. Multifocal images were made using the Z-stack module of AxioVision (Zeiss). Digital montages were created using the "extended depth of field" function of Image-Pro Express 6.0 software (Media Cybernetics, Silver Spring, MD).

Combination of preembedding and postembedding immunocytochemistry for electron microscopy. Three BDA injections were used for electron microscopic observations. In our experimental conditions, postembedding GABA immunogold labeling was not always reliable for quantitative purposes when $\mathrm{DAB}$ was used as a chromogen for the tracer. The DAB precipitate could not be etched properly from the heavily labeled terminals, which increased the chance of identifying a terminal as false GABA negative. Furthermore, fine ultrastructural details were also masked by the electron-dense, homogeneous $\mathrm{DAB}$ precipitate. To overcome this difficulty, the tracer was visualized by the preembedding gold method as described previously (Bokor et al., 2005). Briefly, sections were first incubated with $\mathrm{ABC}$ followed by a signal amplification step using biotinylated tyramide reagent (1:50, 15 min; PerkinElmer Life Sciences, Boston, MA). Next, the sections were incubated with $1 \mathrm{~nm}$ of gold-conjugated streptavidin (1:50; Aurion, Wageningen, The Netherlands) dissolved in TBS containing $0.8 \%$ bovine serum albumin (BSA), $0.1 \%$ gelatin, and $0.05 \%$ Na-azide overnight; postfixed in $2 \%$ glutaraldehyde; and silver intensified with the Aurion R-Gent intensification kit (17-20 min).

All sections were treated with $\mathrm{OsO}_{4}$ ( $1 \%$ for $1 \mathrm{~min}$ and $0.5 \%$ for 20 min, at $4^{\circ} \mathrm{C}$ ), dehydrated in ethanol and propylene oxide, and embedded in Durcupan. During dehydration, the sections were treated with $1 \%$ uranyl acetate in $70 \%$ ethanol for 40 min. Selected blocks containing labeled nigrothalamic terminals were reembedded, 60-nm-thick (silver color) ultrathin sections were cut with an Ultramicrotome (Reichert, Depew, NY), and alternate sections were mounted on copper or nickel grids.

Postembedding GABA immunostaining was performed on nickel grids according to the protocol of Somogyi et al. (1985). The rabbit anti-GABA antibody, kindly donated by Prof. P. Somogyi (Oxford University, Oxford, UK), was raised against GABA bound to BSA. The antibody has been extensively characterized previously (Hodgson et al., 1985; Somogyi et al., 1985). Incubations were performed on droplets of solu- 
tions in humid Petri dishes: $0.5 \%$ periodic acid $\left(\mathrm{H}_{5} \mathrm{IO}_{6}\right)$ for $5 \mathrm{~min}$; wash in distilled water; three times at $2 \mathrm{~min}$ in TBS; $30 \mathrm{~min}$ in $1 \%$ ovalbumin dissolved in TBS; three times at $10 \mathrm{~min}$ in TBS, $1-2 \mathrm{~h}$ in a rabbit antiGABA antiserum (1:3000 in TBS); two times at $10 \mathrm{~min}$ TBS; $10 \mathrm{~min}$ in TBS containing $1 \%$ BSA and $0.05 \%$ Tween $20 ; 2 \mathrm{~h}$ in colloidal goldconjugated goat anti-rabbit IgG [12 nm (Jackson ImmunoResearch, West Grove, PA); 1:20 or 15 nm (GE Healthcare, Little Chalfont, UK); 1:20 in the same solution as before]; two times at 5 min wash in distilled water; 30 min saturated uranyl acetate; wash in distilled water; staining with lead citrate; wash in distilled water. Profiles were considered GABA positive if they contained five times higher density of gold particles than background labeling, as measured above the cell bodies of relay cells. The etching procedure removes the silver precipitate of the tracing (see Fig. $2 A 2, B 2$ ); therefore, only every third grid was reacted for postembedding immunostaining.

Serial electron micrographs were taken using a Megaview II digital camera connected to a Hitachi 7100 electron microscope. For publication, negatives were made using the same electron microscope and scanned at 1600 dpi resolution.

Perfusion: monkey. To minimize the number of animals necessary for this work, the thalami of three adult female macaque monkeys (Macaca mulatta), which received cortical injections in the contralateral hemisphere for other experiments, were used for this study. For perfusion, the anesthesia was induced by intramuscular injection of ketamine hydrochloride $(10 \mathrm{mg} / \mathrm{kg})$, followed by intravenous administration of a lethal dose of sodium pentobarbital. The monkeys were perfused through the heart, first with physiological saline ( $5 \mathrm{~min}, 500 \mathrm{ml}$ ), followed by $400 \mathrm{ml}$ of a fixative containing $2 \%$ paraformaldehyde (TAAB) and $0.5 \%$ glutaraldehyde (TAAB) in acetate buffer ( $\mathrm{pH} 6.0 ; 5 \mathrm{~min}$ ), and finally with 2500 $\mathrm{ml}$ of fixative containing $2 \%$ paraformaldehyde and $0.5 \%$ glutaraldehyde in borate buffer ( $\mathrm{pH} 8.5 ; 90 \mathrm{~min}$ ) (Berod et al., 1981).

After the perfusion, 50- $\mu \mathrm{m}$-thick coronal sections were cut from the anterior part of the thalamus. Alternating sections were processed for type 2 vesicular glutamate transporter (vGLUT2) immunostaining or postfixed in $1 \% \mathrm{OsO}_{4}$, dehydrated, and embedded for electron microscopic analysis. vGLUT2 antibody (1:3000; catalog \#135 102; Synaptic Systems, Goettingen, Germany) was raised against a recombinant glutathione $S$-transferase-fusion protein containing amino acid residues 510 582 of rat vGLUT2. The distribution of vGLUT2 immunolabeling was entirely consistent with previous in situ hybridization and immunocytochemical studies (Fremeau et al., 2001; Land et al., 2004). Following the standard ABC protocol (see above), vGLUT2 was visualized using DABNi. Postembedding GABA immunostaining (described above) was performed on ultrathin sections, cut from the unstained material.

3 D reconstruction and measurements. 3D reconstructions of the bouton membranes, the synapses, puncta adherentia (PA), and the glial sheaths covering the terminals, and in four cases also the mitochondria and the pool of the vesicles, were made using the AnalySIS software (Olympus). Synapses were considered only if the synaptic cleft and the vesicle accumulation were visible on at least two consecutive ultrathin sections.

The membrane of the boutons, the synapses, and glial processes could be identified in more than one ultrathin section, thus the outline of these elements were drawn and connected to the outline of the same structure on the next section. PA, however, were often visible only on one section, because their size was frequently in the range of the thickness of the section. So the puncta outlines were drawn on the section, where they were visible, and duplicated on the next section in each case to the same direction. These two outlines were connected to each other. Without this process, in many cases, puncta would remain two-dimensional objects and could not be visible on the 3D images of the entire boutons. We found that this method was the best approximation to obtain the real appearance of these structures as judged from the occasional tangential view of the puncta network observed when the plane of the section was parallel to the bouton surface.

The volume, the surface, the largest diameter of the reconstructed boutons, and the intersynaptic distances were measured using AnalySIS. The distances between two synapses were measured between the two closest points of the reconstructed synapses. Using these data, the nearest-neighbor synaptic distances and the number of neighboring syn- apses within given distances were also determined. The diameter of each postsynaptic or randomly selected profile was measured on three different sections, and their average was calculated. To measure the area of the synapses, the length of the synapses was determined on each section where a given synapse was visible, summated, and multiplied by the thickness of the sections $(60 \mathrm{~nm})$. The width of synaptic and PA clefts and the presynaptic and postsynaptic density of PA were measured in membranes cut perpendicularly.

To calculate the size and number of synaptic vesicles, 20 -nm-thick sections were cut from two animals, and digital images of tracer-labeled terminals were made at $100,000 \times$ magnification. Because most of the vesicles were elongated, both the long and short diameters of the vesicles were determined. To reduce overestimation of vesicle numbers as a result of double counting vesicles cut on the surfaces and thus present in two consecutive sections, the following method was used. First, clearly identifiable individual vesicles were labeled on each 20 -nm-thick section. Next, the images of consecutive sections were overlaid, and the number of vesicles labeled on both sections were determined and subtracted from the reference section (supplemental Fig. 1, available at www. jneurosci.org as supplemental material). Using this method, we established that $33 \%$ ( $n=1930$ vesicles, 17 pairs of sections) of the vesicles was present in two consecutive sections. Finally, based on the number of remaining vesicles, the vesicle density was determined. The total volume of the vesicle pool was measured in four three-dimensionally reconstructed boutons. The total number of vesicles in the vesicle pool was estimated using the average of the vesicle density data.

Statistics. The diameter distributions of randomly selected dendritic profiles and those contacted by labeled terminals were compared using the $\chi^{2}$ test for homogeneity. The correlations between the number of synapses made by a terminal and its volume, as well as between the number of synapses formed with a given target and the size of the postsynaptic element, were examined in rat and monkey using linear regression. The significance of the correlation was determined by oneway ANOVA using Microsoft (Redmond, WA) Excel. Because most of our data did not show normal distribution, the medians and the interquartile ranges determined by OriginPro 7.5 are shown.

\section{Results}

\section{Injection sites and distribution of nigrothalamic terminals in rats}

SNR axons had dual terminal fields in the thalamus as described previously (Beckstead et al., 1979; Deniau et al., 1992; Sakai et al., 1998). The majority of the terminals were localized to the ventromedial (VM) nucleus, whereas another contingent of fibers innervated the intralaminar nuclei (centrolateral and paracentral nuclei) and the paralamellar part of the mediodorsal nucleus (Fig. $1 B-G$ ). All terminal fields consisted of a dense central core surrounded by scattered terminals. At the light microscopic level, individual nigrothalamic axons had medium- to large-sized terminals, which were distributed unevenly along the axon (i.e., the intervaricose intervals were highly variable).

Double labeling for the tracers and calbindin was used to characterize neurochemically the nigro-recipient thalamic territories. In each case examined $(n=10)$, the nigrothalamic terminals were restricted to the calbindin-positive territories of the VM (Fig. $1 B-I)$ and did not encroach into nearby calbindin-negative areas. Nigrothalamic terminals remained within the limits of calbindin-positive zones in the intralaminar nuclei as well.

\section{Ultrastructural features of rat nigrothalamic terminals}

In the following description, "synapse" refers to a circumscribed membrane specialization including the presynaptic and postsynaptic densities, the vesicles, and the cleft (supplemental Fig. 2, available at www.jneurosci.org as supplemental material). Synaptic specializations, which appeared noncontiguous after $3 \mathrm{D}$ reconstructions, were treated as separate synapses. PA [also known 


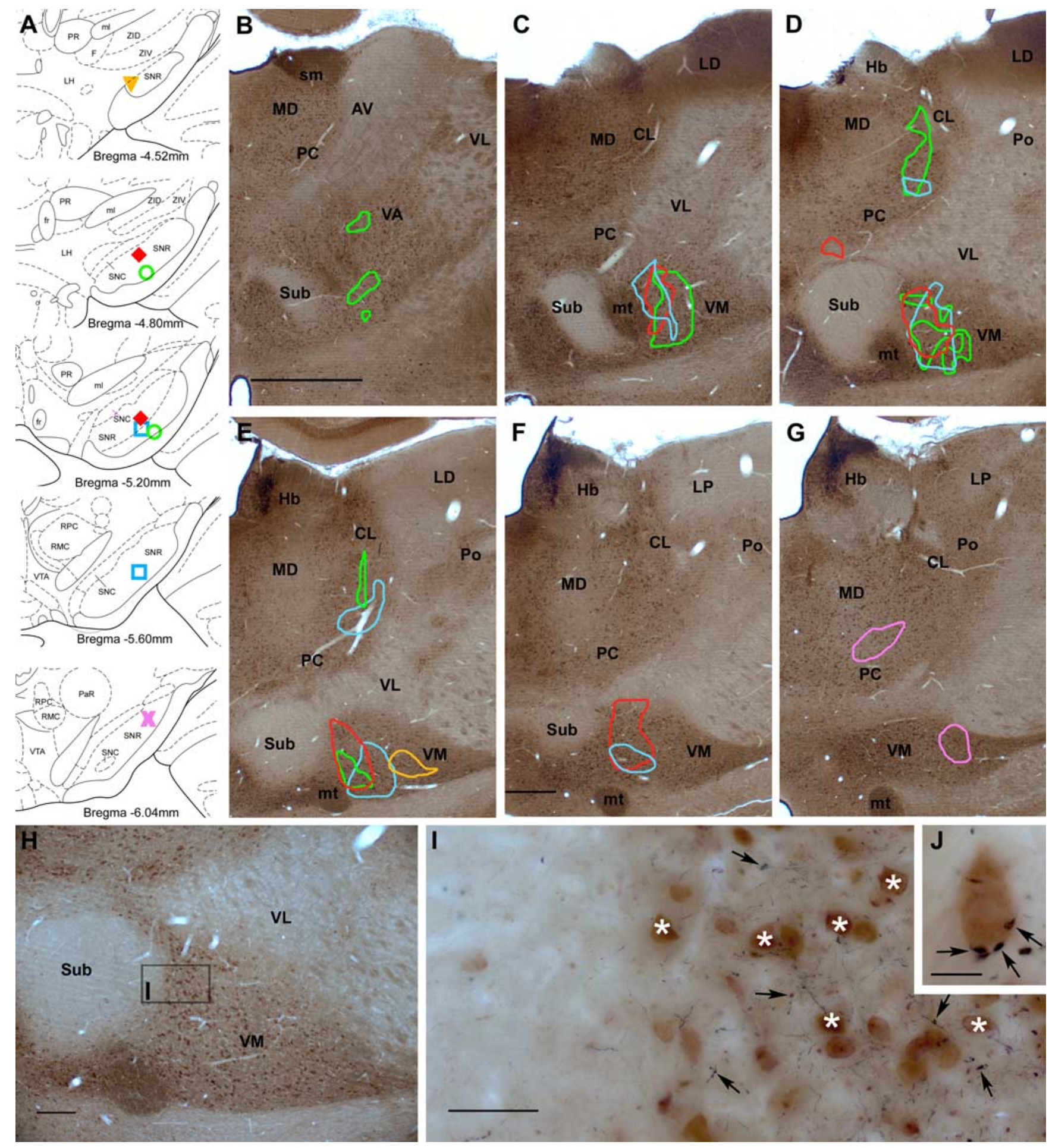

Figure 1. Topography of nigrothalamic terminals and their relationship to calbindin immunostaining in the rat. $A$, Schematic labeling of five injection sites in the SNR shown at five coronal levels of the atlas of Paxinos and Watson (1998). B-G, Distribution of the dense core of nigrothalamic terminal fields in the thalamus, color coded according to the labeling in $A$. Scattered fibers are omitted for clarity. The outlines of the terminal fields are shown overlaid on calbindin-immunostained coronal sections of the thalamus sampled $300 \mu \mathrm{m}$ apart. Note the dual terminal fields in the ventromedial and intralaminar nuclei and the long anteroposterior extent of the terminal fields. Caudal SNR injections result in more dense intralaminar fiber plexuses, whereas rostral injections label only few fibers in these nuclei. $\boldsymbol{H}, \boldsymbol{I}$, Light microscopic images of the VM double stained for BDA (black) and calbindin (brown) after injection of the tracer into SNR. The boxed area in $\boldsymbol{H}$ is shown enlarged in $I$. Nigrothalamic terminals (arrows in $I$ ) are restricted to the zone containing calbindin-immunoreactive cells (asterisks). $\boldsymbol{J}$, Nigrothalamic terminals establish multiple contacts (arrows) on the soma of a calbindin-positive relay cell. I and $\boldsymbol{J}$ are multifocal images. Scale bars: $\boldsymbol{B}-\mathbf{G}, 1 \mathrm{~mm} ; \boldsymbol{H}, 200 \mu \mathrm{m} ; \boldsymbol{I}, 50 \mu \mathrm{m} ; \boldsymbol{J}, 10 \mu \mathrm{m}$. AV, Anteroventral thalamic nucleus (n.); $\mathrm{CL}$, centrolateral thalamic n.; $F$, nucleus of the fields of Forel; fr, fasciculus retroflexus; $H b$, habenular n.; LD, laterodorsal thalamic n.; $L$, lateral hypothalamic area; $L P$, lateral posterior thalamic $n$.; $M D$, mediodorsal thalamic n.; ml, medial lemniscus; mt, mammillothalamic tract; PaR, pararubral nucleus; PC, paracentral thalamicn.; PR, prerubral field; Po, posterior thalamic n. group; RMC, red nucleus, magnocellular part; $\mathrm{RPC}$, red nucleus, parvicellular part; $\mathrm{sm}$, stria medullaris of thalamus; SNC, substantia nigra, compact part; Sub, submedius thalamic n.; VA, ventral anterior thalamic $\mathrm{n}$.; VL, ventrolateral thalamic $\mathrm{n}$.; VTA, ventral tegmental area; ZID, zona incerta dorsal part; ZIV, zona incerta ventral part. 
as filamentous contacts (Lieberman and Spacek, 1997)] are distinct from synapses, in that they have thick, asymmetric membrane specialization and lack vesicle accumulation, as described previously (supplemental Fig. 2, available at www. jneurosci.org as supplemental material) (Lieberman and Spacek, 1997).

In the VM of three rats, altogether 58 anterogradely labeled profiles with a synapse(s) were followed in serial electron microscopic sections. Two of these proved to be intervaricose axonal segments with a synapse. Of the remaining 56 nigrothalamic boutons, 34 were followed in serial sections from end to end, whereas in the case of 22, only segments of the boutons were analyzed. Of the 34 complete terminals, the membranes were reconstructed for volume analysis in 29 cases. Of these 29 terminals, the quality of the membranes allowed to examine the exact number and position of the synapses throughout the entire bouton in 22 cases (for a summary of all boutons, see supplemental Table 1, available at www.jneurosci.org as supplemental material).

Nigrothalamic terminals in the VM were medium to large sized (see below for details) and contained a large number of mitochondria and flattened or pleomorph vesicles. Multiple synapses were frequently evident even in single sections (Fig. 2). The terminals were also characterized by a large number of PA and by a glial envelope around the membrane surface not apposed to the postsynaptic cell (Fig. 2). When the plane of the section was parallel to the opposing membranes, occasionally a latticelike structure consisting of a network of PA could be observed on the membrane surfaces (data not shown). The median widths of the electron-dense plaques on the presynaptic and the postsynaptic side of PA were 24.19 versus $42.00 \mathrm{~nm}$ (interquartile ranges of $20.99-27.78$ and $36.62-47.88 \mathrm{~nm}$, respectively; $n=38$ ). In contrast, the presynaptic and postsynaptic densities of the synapses were negligible. Widening of the gap between the membranes was evident at both the synapses and the PA. The difference in the width of the synaptic clefts (median, $20.24 \mathrm{~nm}$; interquartile range, $18.37-21.16 \mathrm{~nm} ; n=36$ ) and PA clefts (median, $17.75 \mathrm{~nm}$; interquartile range, $14.77-19.15 \mathrm{~nm}$; $n=38$ ), although statistically significant (Mann-Whitney $U$ test, $p<0.05$ ), cannot be used as a distinguishing feature because of the large overlap in the values.

The GABA content of the nigrothalamic VM terminals was examined in 54 cases after postembedding GABA-gold immunostaining. All of the entirely reconstructed 34 terminals were GABA positive (Fig. 2A2,B2). Of the 20 partially reconstructed terminals, 17 were GABA positive, whereas in the remaining three cases, the GABA content could not be unequivocally identified.

Six nigrothalamic terminals were examined in the paracentral nucleus as well. Their ultrastructural features were identical to
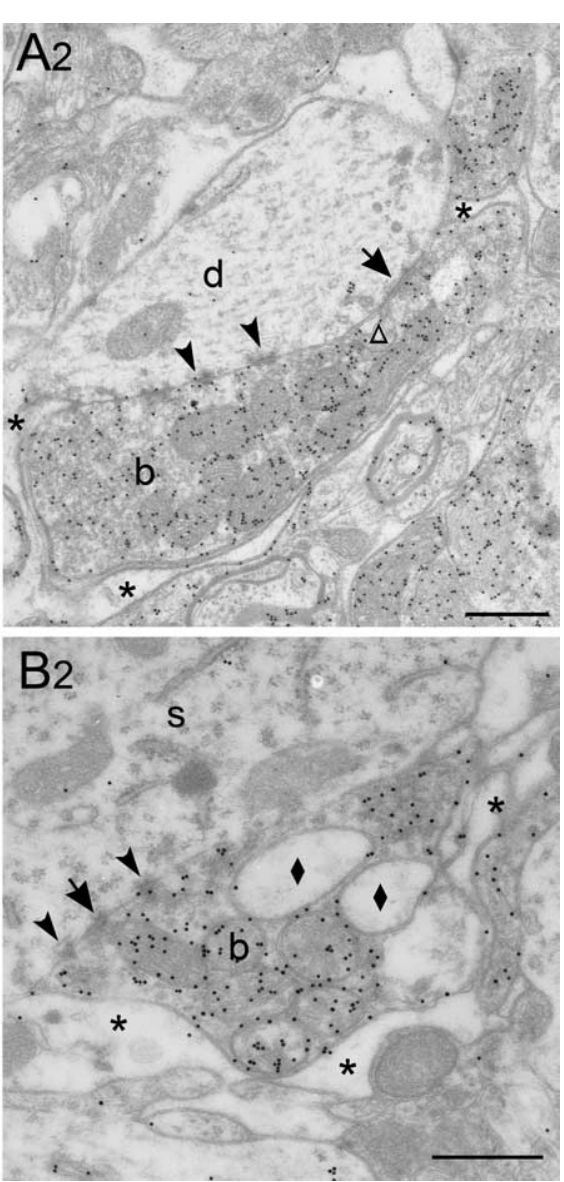

Figure 2. Ultrastructural features of nigrothalamic terminals in the VM of rat. High-power electron microscopic images. particles (thin arrows) indicate the anterograde tracer. $\mathbf{A 2}, \mathbf{B} \mathbf{2}$, Small black particles are the results of postembedding GABA mmunostaining. Note that in $\boldsymbol{A} \mathbf{2}$ and $\mathbf{B} \mathbf{2}$, the anterograde signal is absent because of the etching procedure of the postembedntia (arrowheads) with their postsynaptic targets [proximal dendrite (d) in $\boldsymbol{A}$; soma (s) in $\boldsymbol{B}$ ]. Note that vesicles are present outside the clusters of mitochondria. The outer surfaces of the terminals are covered by glial sheets (asterisk). Glia can intrude into the synapse-bearing surface $(\boldsymbol{A} \mathbf{1}, \boldsymbol{A} \mathbf{2}$, triangles) or into the terminal (B1, $\boldsymbol{B} \mathbf{2}$, diamonds). Scale bars, $0.5 \mu \mathrm{m}$.

the VM terminals (supplemental Fig. 3, available at www.jneurosci.org as supplemental material).

\section{Target selectivity of rat nigrothalamic terminals}

The type (soma, dendrite) and the minor diameters of the profiles postsynaptic to nigrothalamic terminals $(n=56)$ were examined in VM and compared with a random sample $(n=$ 94) from the same nucleus (Fig. $3 A$ ). In the case of the random sample, not only the number of dendrites was plotted but also the summated perimeter of the dendrites, which better reflects the available potential synaptic membrane surface (Fig. 3A). The minor diameter of the vast majority $(84 \% ; n=79)$ of dendrites in the random sample was below $0.8 \mu \mathrm{m}$, indicating that the neuropil mainly contains thin, higher-order dendrites. In contrast, among the dendrites targeted by nigrothalamic terminals, only $16 \%(n=9)$ were below $0.8 \mu \mathrm{m}$. The majority of the SNR inputs $(77 \% ; n=43)$ contacted dendrites thicker than $0.8 \mu \mathrm{m}$, which were comparably rare in the neuropil. Seven percent $(n=4)$ of the terminals innervated somata. The difference between the distribution of dendritic diameters in the target and random sample was statistically highly significant $\left(\chi^{2}\right.$ test, $\left.p<0.001\right)$. 


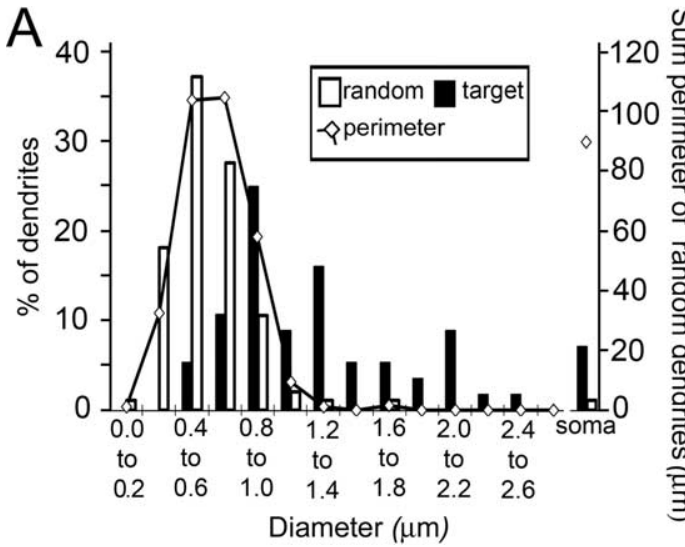

Figure 3. Target selectivity and synapse size of the rat nigrothalamic terminals. $A$, Bar graph showing the minor diameter of randomly selected dendrites and the target dendrites of nigrothalamic terminals in the VM using $0.2 \mu \mathrm{m}$ bin width. The distribution of the diameter of random dendrites is shown in two ways, as bars representing the percentage of dendrites in each bin (similar to the target dendrites; left $y$-axis) but also as a continuous line showing the summated perimeter of the dendrites in each bin (right $y$-axis). The first one is used for statistical comparison, whereas the second one better represents the available target surfaces. Note that the distribution of the target diameters is skewed toward the larger values compared with random sample. $\boldsymbol{B}$, Whisker plot showing the size distribution of nigrothalamic synapses in the VM. max, Maximum; min, minimum.

\section{D reconstruction of nigrothalamic terminals}

All 34 nigrothalamic terminals followed from end to end in serial electron microscopic sections established symmetrical synaptic contacts with their target elements via multiple, distinct synapses (Fig. 4 and supplemental movie 1, available at www.jneurosci.org as supplemental material). The median number of synapses established by a single terminal was $8.5(n=22$; minimum to maximum, 2-26; interquartile range, 6-12) (for a summary of quantitative data, see supplemental Table 2, available at www. jneurosci.org as supplemental material). In each case, all synapses of a single terminal converged on a single postsynaptic target. The surface area of the synaptic specializations showed great heterogeneity. A larger than fourfold difference was found between the smallest $\left(0.014 \mu \mathrm{m}^{2}\right)$ and largest $\left(0.062 \mu \mathrm{m}^{2}\right)$ synapses (median, $0.029 \mu \mathrm{m}^{2}$; interquartile range, $0.021-0.042 \mu \mathrm{m}^{2} ; n=39$ ) (Fig. $3 B)$.

The volume of the nigrothalamic boutons displayed large variability (median, $1.76 \mathrm{\mu m}^{3}$; minimum to maximum, 0.32 $6.06 \mu \mathrm{m}^{3}$; interquartile range, $\left.1.29-3.64 \mu \mathrm{m}^{3} ; n=29\right)$. The long diameter of the terminals was most frequently in the range of 2-4 $\mu \mathrm{m}$ (median, $2.77 \mu \mathrm{m}$; interquartile range, 2.37-3.43 $\mu \mathrm{m} ; n=$ 29). The surface of the bouton membrane varied between 4.17 and $26.77 \mu \mathrm{m}^{2}$ (median, $12.46 \mu \mathrm{m}^{2}$; interquartile range, $8.76-$ $\left.14.49 \mu \mathrm{m}^{2} ; n=24\right)$. In two cases, the ratio of the total bouton surface per synapses surface was determined. In these cases, the summated surfaces of the synapses constituted 2.74 and $2.62 \%$ of the total bouton surface.

$3 \mathrm{D}$ reconstruction of the membranes of the terminals apposing their postsynaptic targets revealed a common principle in the arrangement of synapses and PA. The vast majority of the synapses were situated at the outer part of the opposing membrane surfaces in a circular manner, whereas the middle part was occupied by a network of PA (Fig. 4 and supplemental movie 1, available at www.jneurosci.org as supplemental material). Certain PA were localized close to the edges of the synapses or outside the circle of the synapses.

In 22 of the 34 cases, the synapse-bearing surface of the terminals formed one plane, and the synapses were established on shafts or soma membranes. In the remaining 12 cases, one or two of the synapses were formed on the dendritic appendages emarest of the synapses. In one case, the glia separated four synapses from the remaining seven (Fig. 4A).

Mitochondria were packed as a dense cluster in the central part of the boutons within the terminals (Fig. 4B2,B4). Very few vesicles could be found among them or in the space between the mitochondria and the network of PA (supplemental Fig. 2D-F, available at www.jneurosci.org as supplemental material). Vesicles also avoided the spaces where the mitochondria were too close to the bouton membrane. Large, homogenous pools of vesicles filled the outer, mitochondria-free part of the terminals (Fig. 4B2 and supplemental Fig. 1, available at www.jneurosci.org as supplemental material). Their circular arrangement corresponded to the distribution of synapses. Each synapse was characterized by vesicle accumulation and weakly electron-dense filamentous projections extending into the cytoplasm (Fig. 4A5-A8 and supplemental Fig. 2A$C, F$, available at www.jneurosci.org as supplemental material). Most of the vesicles were elongated. The median length of their long and short axis were $31.43 \mathrm{~nm}$ (interquartile range, $29.08-34.39 \mathrm{~nm}$ ) and $15.22 \mathrm{~nm}$ (interquartile range, 12.47$19.44 \mathrm{~nm})$, respectively.

The proportion of bouton volume occupied by the vesicle pool was determined in four cases. Vesicles occupied approximately one-quarter of the bouton volume $(22.33,23.11,23.73$, $26.85 \%$; median, $23.42 \%$ ).

To gain an estimate of the entire number of vesicles within a terminal, the density of vesicles were determined in a given volume using serial 20-nm-thick sections (see Materials and Methods and supplemental Fig. 1, available at www.jneurosci.org as supplemental material). The median density of vesicles was 18,785 vesicles $/ \mu \mathrm{m}^{3}$ (interquartile range, 12,599-20,634 vesicles $/ \mu \mathrm{m}^{3} ; n=7$ stacks of serial sections, 17 pairs of sections). According to this, the total number of vesicles in the four terminals where the volume of vesicle pool was established is between 5148 and 9548 (the volume of these boutons was between 1.15 and $2.28 \mu \mathrm{m}^{3}$ ).

The exact number of vesicles in the synapse, attached to the presynaptic membranes or in its immediate vicinity could not be determined even using the 20 -nm-thick sections and $100,000 \times$ magnification (supplemental Fig. 1, available at www. jneurosci.org as supplemental material). According to our rough 

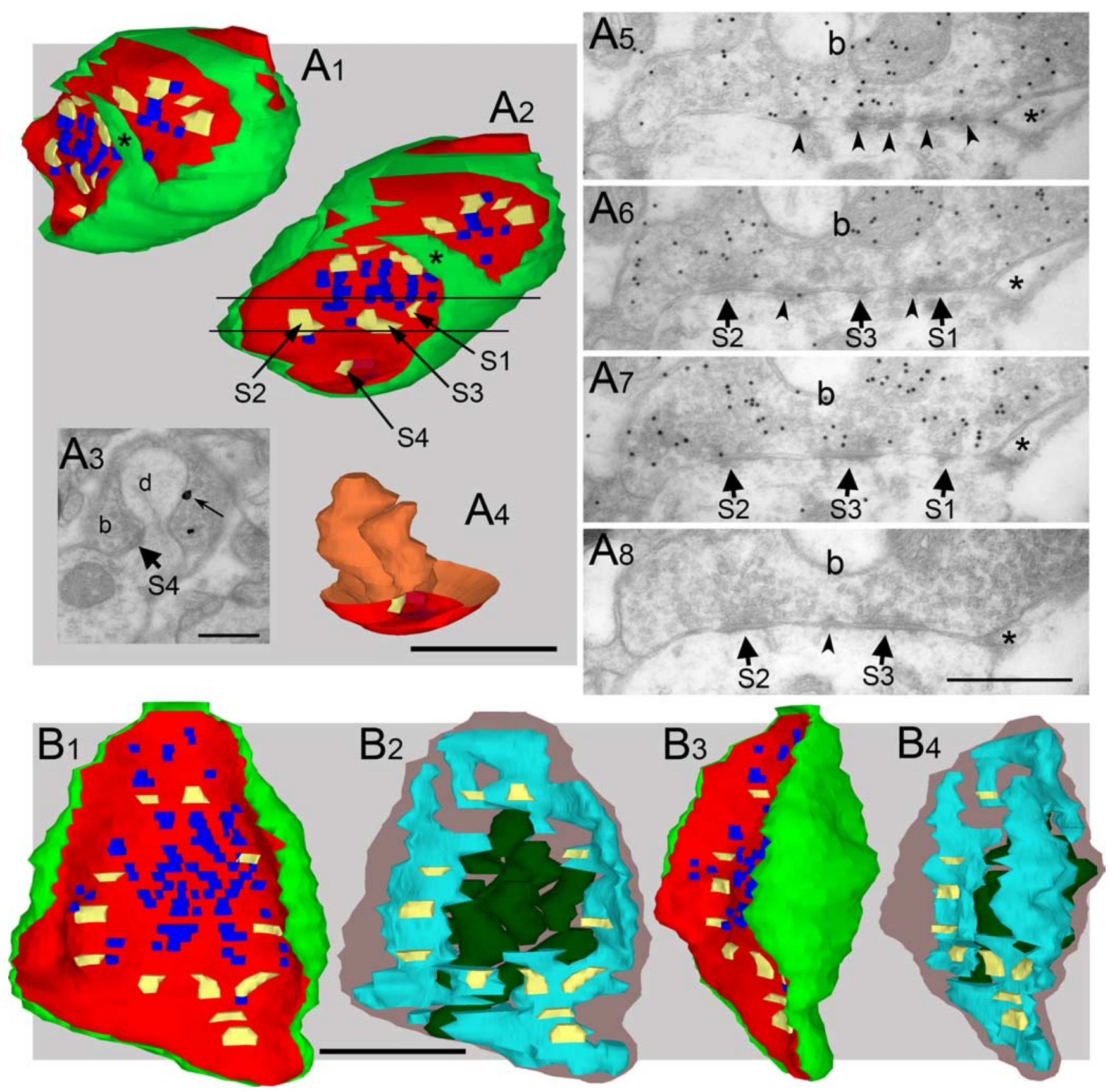

Figure 4. 3D reconstructions of nigrothalamic terminals in rats. A1, A2, Two views of the same terminal. Yellow, Synapses; blue, PA; red, membrane of the terminal; green, glia. All synapses are located on the same side of the terminal and contact the same postsynaptic target (not shown). A glial process intrudes into the synapse-bearing surface (asterisks), but no glia is apparent among the majority of the synapses. $A 3$, A synapse ( $\$ 4$ arrow) is established on a dendritic appendage of the target dendrite, which protrudes into the terminal. Thin arrow, Silver-intensified gold particle indicating the tracer. A4, 3D reconstruction of the dendritic appendage (orange). A5-A8, Synapses (S1-S3 arrows) between the two black lines in $A 2$ are shown in serial electron microscopic sections. Sections shown in A5-A7 were postembedding immunoreacted for GABA. Arrowheads indicate PA. A1-B4, Two views of the outer surface (B1-B3; same color coding as above) and the inner organelles ( $\boldsymbol{B} 2-\boldsymbol{B}$; ; blue, vesicle pool; dark green, mitochodria) of another nigrothalamic terminal. Note the rim of synapses around the central meshwork of PA in $\boldsymbol{B} 1$ and the corresponding

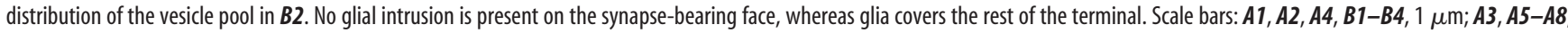
$0.5 \mu \mathrm{m}$.

estimate, $\sim 15-30$ vesicles can be found within a $60 \mathrm{~nm}$ range around at each synapse.

Serial reconstruction revealed that more than one nigrothalamic terminal of the same axon can innervate the same dendrite (supplemental Fig. 3, available at www.jneurosci.org as supplemental material). Systematic analysis of the number of terminals per target is beyond the scope of the present account.
Ultrastructural features of large GABAergic terminals in the nigro-recipient thalamus of macaques

Previous data demonstrated that nigrothalamic terminals can be unequivocally identified in the macaque monkey based on the following observations. (1) The only synaptic population in the somata and first-order dendrites of relay cells and the vast majority of the boutons contacting second-order dendrites originate in 

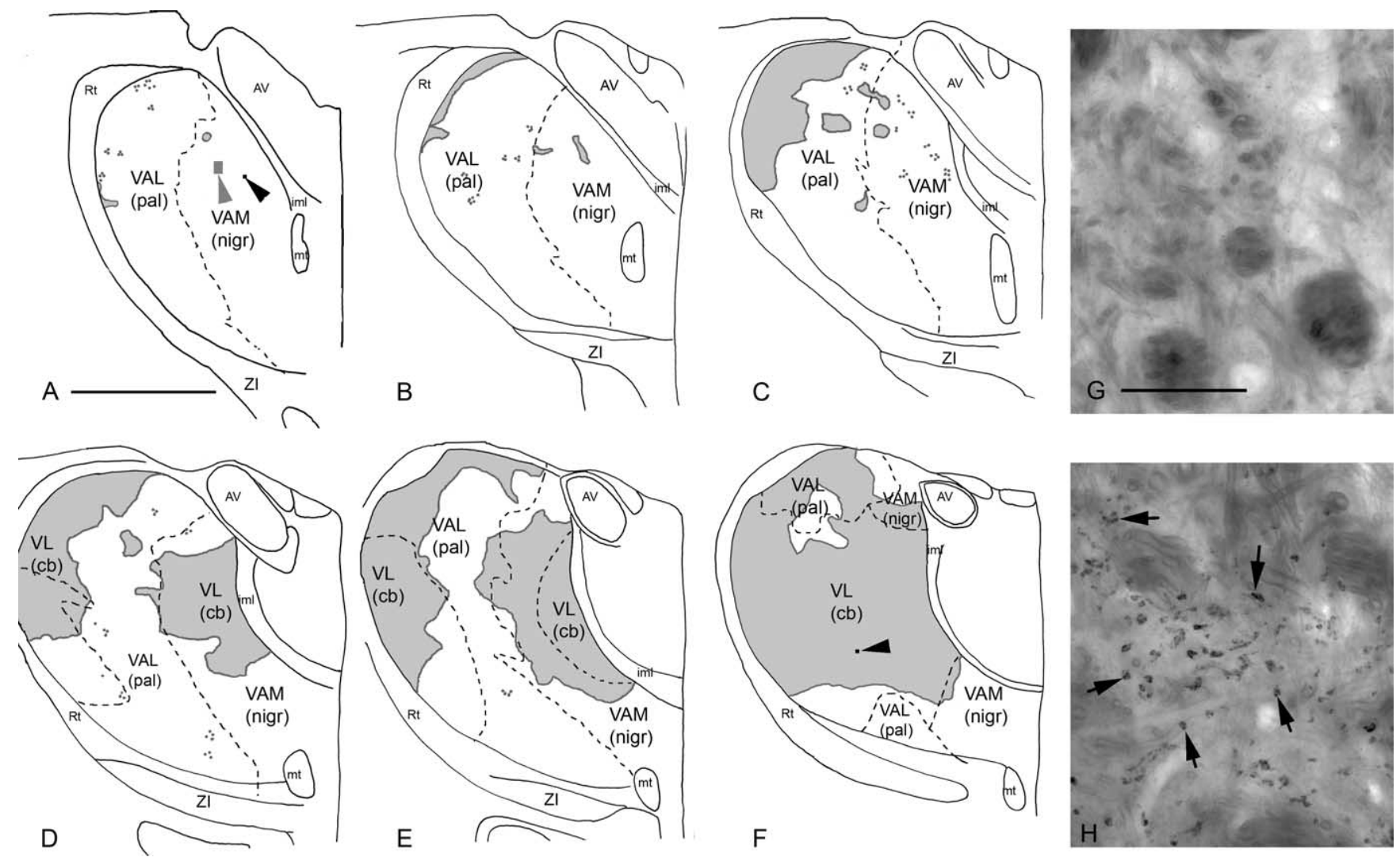

Figure 5. Distribution of VGLUT2 immunostaining in the motor thalamus of the macaque monkey. $\boldsymbol{A}-\boldsymbol{F}$, The gray area shows thalamic regions containing large vGLUT2-immunoreactive terminals on the schemes modified from the atlas of Paxinos et al. (2000), which depict the outlines of the three large, nonoverlapping regions of the motor thalamus: cerebellar-recipient (cb), nigro-recipient (nigr), and pallido-recipient (pal). The panels are in a coronal plane in rostral $(\boldsymbol{A})$ to caudal $(\boldsymbol{F})$ order. Note the general correspondence between the vGLUT2-immunoreactive regions (gray) and the cb areas. A significant discrepancy is visible only in the dorsolateral part in $\boldsymbol{C}$ and $\boldsymbol{D}$. Note the vGLUT2-positive islands in $\boldsymbol{B}$ and $\boldsymbol{C}$, which is in line with cerebellar tracing studies. The gray arrowhead in $\boldsymbol{A}$ points to the region reembedded for electron microscopic analysis. Black arrowheads in $\boldsymbol{A}$ and $\boldsymbol{F}$ indicate the positions of light microscopic images shown in $\boldsymbol{G}$ and $\boldsymbol{H}$, respectively. $\boldsymbol{G}$, $\boldsymbol{H}$, High-power, multifocal light microscopic images of vGLUT2 immunostaining in the nigro-recipient $(\boldsymbol{G})$ and cerebello-recipient $(\boldsymbol{H})$ thalamus. Large vGLUT2-positive terminals are entirely absent in nigrorecipient territories, whereas they are abundant in the cerebellar zone (arrows). Scale bars: $\boldsymbol{A}-\boldsymbol{F}, 2 \mathrm{~mm} ; \mathbf{G}, \boldsymbol{H}, 50 \mu \mathrm{m}$. mt, Mammillothalamic tract; AV, anteroventral thalamic nucleus; Zl, zona incerta; Rt, reticular thalamic nucleus; iml, internal medullary lamina; VAL, ventral anterior thalamic nucleus, lateral part; VAM, ventral anterior thalamic nucleus, medial part; VL, ventrolateral thalamic nucleus.

SNR (Ilinsky and Kultas-Ilinsky, 1990; Kultas-Ilinsky and Ilinsky, 1990). The diameter of these dendrites is thicker than $1 \mu \mathrm{m}$ (Ilinsky and Kultas-Ilinsky, 1990). (2) The GABA-positive nigrothalamic terminals are characterized by large-size, multiple PA; numerous mitochondria; and lack of postsynaptic specialization (Kultas-Ilinsky and Ilinsky, 1990). These features clearly distinguish them from the other two types of vesicle-containing GABAergic elements of the primate thalamus (i.e., interneuron dendrites and terminals from the reticular thalamic nucleus) (Ohara and Lieberman, 1993; Ilinsky et al., 1999).

In primates, nigro-recipient territories are well separated from areas receiving pallidal and cerebellar inputs, with minimal or no overlap (Tracey et al., 1980; Ilinsky et al., 1985; Ilinsky and Kultas-Ilinsky, 1987; Rouiller et al., 1994; Percheron et al., 1996; Sakai et al., 1996). However, the anterior border of the cerebellar thalamus is difficult to delineate in coronal sections (Ilinsky and Kultas-Ilinsky, 1987). In this study, vGLUT2, the well established marker of subcortical excitatory afferents (Fremeau et al., 2001; Land et al., 2004; Lavallee et al., 2005), was used in combination with the atlas of Paxinos et al. (2000) to draw the border of nigro-recipient zones (Fig. 5). vGLUT2 immunostaining in the macaque thalamus labeled large terminals with the known feature of driver afferents (Fig. $5 H$ ). The distribution of vGLUT2-positive terminals in the anterior (motor) portion of the thalamus closely matched the terri- tories occupied by cerebellar afferents described in previous tract tracing studies (Kalil, 1981; Asanuma et al., 1983; Berkley, 1983; Percheron et al., 1996; Ilinsky et al., 1997) (Fig. $5 A-F$ ). In the deep cerebellar nuclei, the parent cell bodies of the thalamic vGLUT2containing axons also proved to be vGLUT2 immunopositive, confirming the use of vGLUT2 as a marker for cerebello-recipient thalamic territory in the motor thalamus (data not shown). For the electron microscopic analysis, the nigrothalamic block was reembedded rostral to the most anterior vGLUT2-positive patches close to the internal medullary lamina, to ensure the avoidance of both pallidal and cerebellar territories (Fig. 5A).

Based on the criteria listed above, we examined GABAergic terminals contacting the somata and thick dendrites of relay cells in the nigro-recipient zone of three macaque monkeys. For quantification, the animal with the best ultrastructure was selected, but the results were qualitatively assessed in the other two monkeys as well. Eighteen GABAergic terminals were examined in serial sections, of which 2 contacted somata, 14 contacted dendrites thicker than $1 \mu \mathrm{m}$ (average minor diameter, $1.71 \mu \mathrm{m}$; minimum to maximum, $1.06-2.12 \mu \mathrm{m}$ ), and 2 contacted thinner dendrites $(0.72$ and $0.78 \mu \mathrm{m})$. Ten terminals were completely and eight were partially reconstructed. Confirming previous results (Ilinsky and Kultas-Ilinsky, 1990; Kultas-Ilinsky and Ilinsky, 1990), all GABAergic boutons contacting the somata and dendrites in our 
sample displayed the ultrastructural features of nigrothalamic terminals (Fig. 6). The ultrastructural characteristics of these terminals were similar to rat nigrothalamic boutons (see below).

\section{Comparison of the 3D ultrastructure of the rat and macaque terminals}

$3 \mathrm{D}$ reconstruction revealed that similar to SNR boutons of rats, all macaque GABAergic terminals in our sample established multiple synapses on postsynaptic GABA-negative relay cells (Fig. 7 and supplemental Table 2, available at www. jneurosci.org as supplemental material). The average number of synapses/terminal (minimum to maximum, 5-12; median, 8.5 ; interquartile range, $7-10 ; n=10$ ) were surprisingly similar to the rat data (median, 8.5; for quantitative comparison of rat and monkey data, see supplemental Table 2, available at www.jneurosci.org as supplemental material). In 8 of the 10 macaque terminals analyzed, all synapses were established on a single target, like in rat. Two terminals, however, also contacted very thin dendrites (Fig. 6A1,B1) with two synapses on each (Fig. 7B2). This second postsynaptic element proved to be a GABA-positive interneuron dendrite in one case (Fig. 6B1). The GABA content of the dendrite in the other case could not be established.

The arrangement of synapses and PA of the macaque terminals replicated the pattern found in rat nigrothalamic boutons (i.e., a centrally located network of PA surrounded by laterally spaced synapses) (Fig. 7 and supplemental movie 2, available at www.jneurosci.org as supplemental material). Glial ensheathment was identified in all 18 cases. In the 10 completely reconstructed terminals, the glial processes covered the entire nonapposing surface. The apposing surface, however, was almost completely free of glia (Fig. 7 and supplemental movie 2, available at www. jneurosci.org as supplemental material), similar to rats. In two cases, synapses were located on a dendritic intrusion.

In the case of the macaque GABAergic terminals, the volume (median, $2.91 \mu \mathrm{m}^{3}$; interquartile range, $2.09-3.78 \mu \mathrm{m}^{3} ; n=$ 10) and the length of the longest diameter (median, $2.96 \mu \mathrm{m}$; interquartile range, $2.62-3.77 \mu \mathrm{m} ; n=10$ ) were in the same range as those of the nigrothalamic terminals of rats (Fig. 8).

The number of synapses showed a strong correlation with the volume of the terminals in rats, whereas the correlation was weaker in the case of monkeys (Fig. $8 \mathrm{~A}$ ). The correlation reached statistical significance only in the case of rat terminals (one-way ANOVA, $p<0.001$ ). The number of synapses per bouton was not correlated with the diameter of the postsynaptic dendrite in either species (Fig. 8B). Terminals with 8-12 synapses contacted arrowhead indicates $P A$.
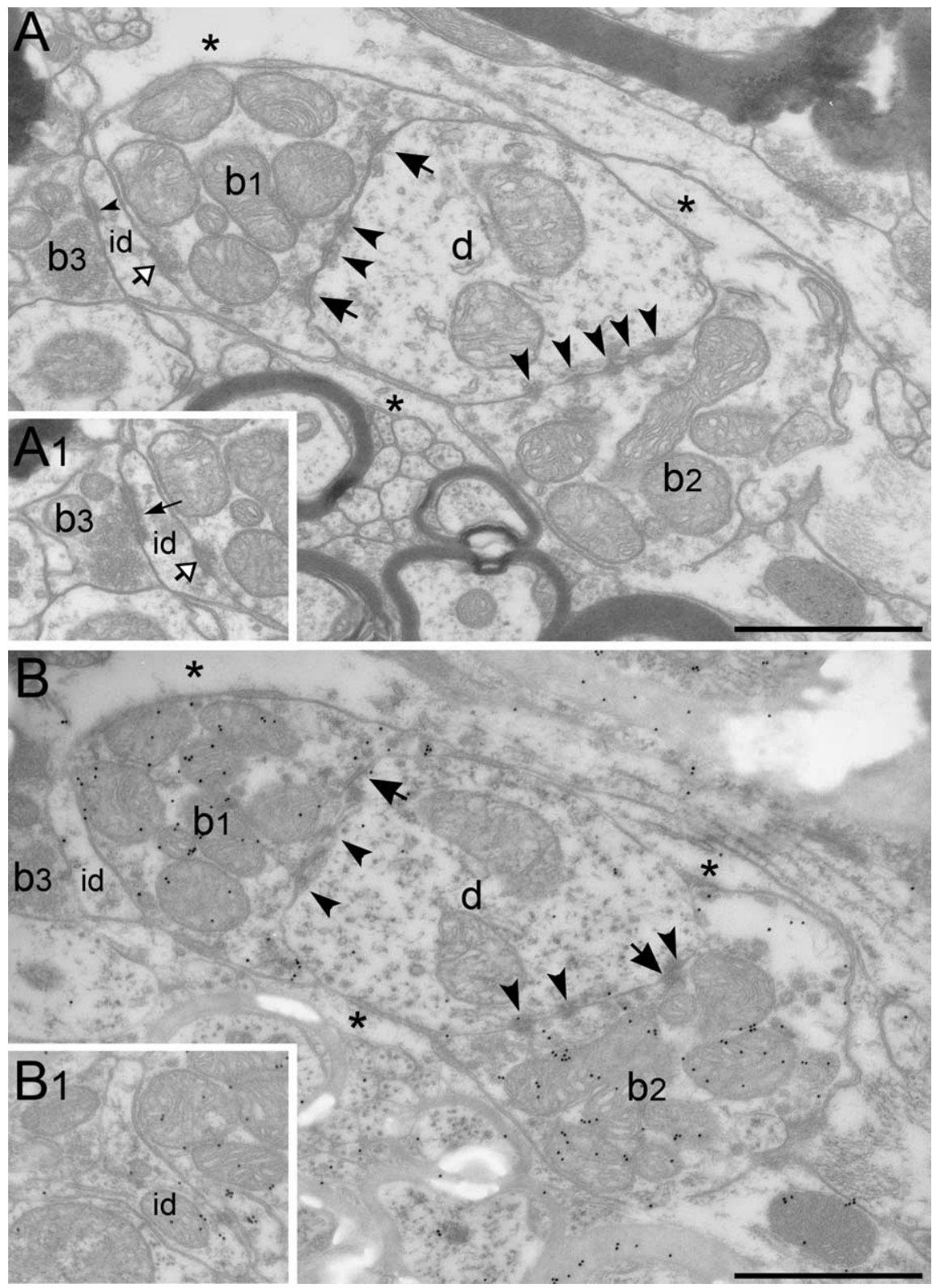

Figure 6. Electron microscopic images of two large GABAergic boutons showing the ultrastructural features of nigrothalamic terminals in monkey. High-power electron microscopic images of two axon terminals (b1, b2) converging on the same largecaliber dendrite in the nigro-recipient part of the macaque thalamus are shown. The neighboring section $(\boldsymbol{B})$ is immunoreacted for GABA. The ultrastructure of the terminals is identical to nigrothalamic terminals described previously in single sections (Kultas-llinsky and llinsky, 1990) (i.e., large-size, numerous mitochondria; several PA; multiple synapses; thick-caliber postsynaptic target; flat vesicles). Besides the main relay cell target, terminal b1 also establishes a synapse $(\boldsymbol{A}, \boldsymbol{A} \boldsymbol{1}$, white arrowheads) on a thin dendritic process (id) identified as an interneuron dendrite on the GABA-immunostained section (B1). This dendrite also receives an asymmetrical synapse ( $\boldsymbol{A} 1$, thin arrow) from a non-GABAergic terminal (b3). Glial sheets around the terminal are labeled with asterisks. A 3D reconstruction of bouton b1 is shown in Figure $7 B$. Scale bar, $0.5 \mu \mathrm{m}$. Black arrow indicates synapse;

dendrites with a diameter ranging from 0.8 to $2 \mu \mathrm{m}$, both in rat and monkey.

\section{Analysis of the intersynaptic distances in rat and monkey}

Transmitter diffusion and spillover among the synapses significantly affect the magnitude and kinetics of postsynaptic responses. Thus, we measured the intersynaptic distances in the $3 \mathrm{D}$ reconstructed rat and monkey terminals (rat: $n=4$ terminals, 38 synapses, 188 intersynaptic distances; monkey: $n=4$ terminals, 33 synapses, 122 intersynaptic distances). 

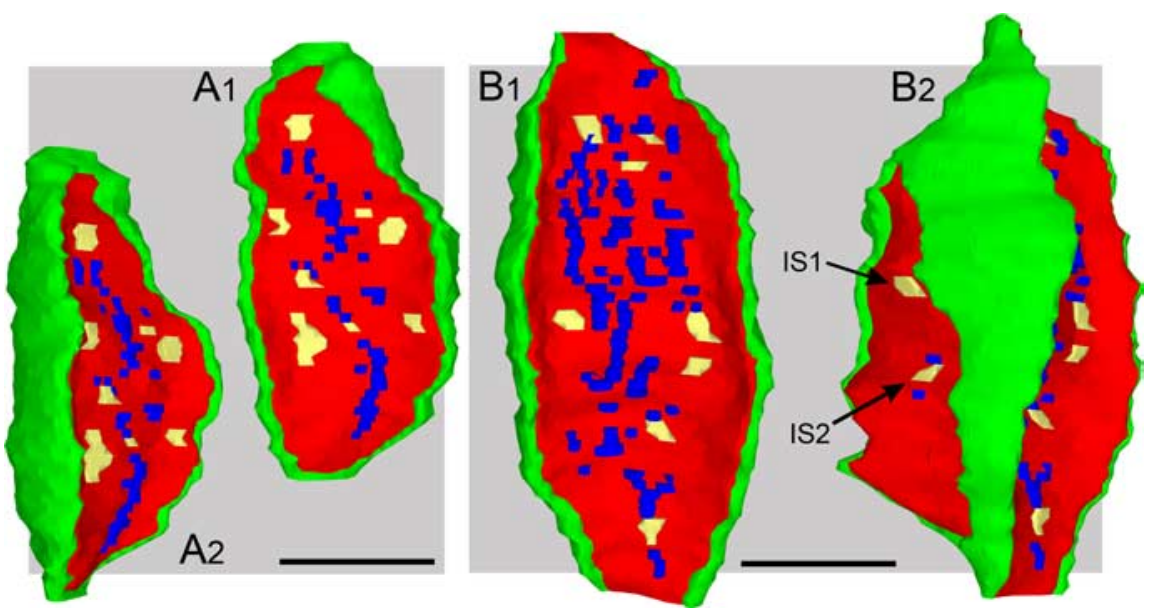

Figure 7. 3D reconstructions of large GABAergic boutons showing the ultrastructural features of nigrothalamic terminals in monkey. Two terminals are shown. Color coding is as in Figure 4.A1, A2, Two views of the same GABAergicterminals. All synapses contacting the relay cell dendrite (not shown) are located on the same side of the terminal. Note the centrally located network of PA and the laterally placed synapses, similar to the arrangement found in rat nigrothalamic terminals (Fig. 4). B1, B2, Two views of another terminal (b1 terminal on Fig. 6). In this case, besides the eight synapses (B1, yellow) contacting a relay cell dendrite, the opposite side of the terminal establishes two other synapses (IS1, IS2; one of them is shown in Fig. 6, $A$ and $A 1$ ) on an interneuron dendrite. 3D reconstruction of the terminal in $A$ is shown in supplemental movie 2 (available at www.jneurosci.org as supplemental material). Scale bars, $1 \mu \mathrm{m}$.

One synapse of a rat terminal, which was separated from the rest by a glial process, was not included in this analysis.

The data were analyzed in three ways. First, all the synaptic distances of the four rat and four monkey terminals were measure and compared. This analysis examined the overall spatial relationship of the synapses relative to each other. The median value of all intersynaptic distances was highly similar in rats $(900 \mathrm{~nm}$; interquartile range, $529-1189 \mathrm{~nm}$ ) and monkeys (796 nm; interquartile range, 480-1305 nm) (Fig. 9A).

Next, we examined the average number of synapses within increasing distances from any given synapse (Fig. 9C). This allows the estimation of the number of synapses reached by the transmitter once the diffusion parameters are established or modeled. The rat and monkey data showed very close correspondence. The average number of synapses within $0.5 \mu \mathrm{m}$ was 1.94 in rats and 2.11 in monkeys; the same values for $0.75 \mu \mathrm{m}$ were 3.83 versus 3.46 synapses and for $1 \mu \mathrm{m}$ were 5.42 and 4.46 synapses in rat versus monkey, respectively, demonstrating that the position of the synapses relative to each other is highly similar in the two species. These data are further justified if nearest-neighbor synaptic distances are examined (Fig. 9D). The median values of the nearest-neighbor distances between synapses were $169 \mathrm{~nm}$ (interquartile range, 92-244) in the case of the rat and $178 \mathrm{~nm}$ (interquartile range, 123-253) in the monkey. The cumulative curves of the nearest-neighbor distances almost entirely overlapped (Fig. 9D). In both species, $80 \%$ of the nearest-neighbor distances were below $300 \mathrm{~nm}$.

\section{Discussion}

Our quantitative 3D electron microscopic data demonstrate that all rodent and primate nigrothalamic terminals establish multiple synaptic contacts on a single postsynaptic target with closely spaced synapses, which are not separated by glial processes. To our knowledge, this is the first 3D characterization of a GABAergic pathway with exclusively multisite terminals in the forebrain. Because of the size of the terminals and the arrangement of the active zones, this GABAergic bouton type can serve as a new model system to study transmitter diffusion and spillover among synapses. The active zones are on a flat surface; thus the kinetics of transmitter movement can be easily modeled.

The morphology of nigrothalamic terminals: primate-rodent comparison Our data are consistent with the previous electron microscopic description of rat and monkey SNR terminals in the thalamus, which were based on single-section analysis (Kultas-Ilinsky and Ilinsky, 1990; Sakai et al., 1998; Tsumori et al., 2002). The statistical comparison with random sample clearly demonstrated preferential innervation of thick dendrites in the VM.

$3 \mathrm{D}$ features of rat and monkey SNR terminals showed surprising similarity both in qualitative and quantitative terms. The number of synapses and their spatial arrangement relative to each other showed closely matching values. There is an extensive debate in the literature as to what extent rodent data are applicable to primate conditions. Indeed, because of the difference in the organization of basal gangliathalamus circuits in the two species, many researchers dismiss rodent data as irrelevant for, for example, Parkinson's disease research. Our data demonstrate that the major morphological features of the SNR terminals, and thus probably the mode of GABAergic transmission associated with it, is conserved in the rodent and primate lineages despite the different organization of the motor thalamus in these two mammalian orders. This strongly suggests that in pathophysiological studies the rodent nigrothalamic pathway can serve as model for the primate condition.

\section{Multisite boutons and transmitter spillover}

The main question is why multisite boutons are favored instead of multiple single-site terminals? The most plausible answer is that multisite boutons allow closely spaced synapses. In both species studied here, the nearest-neighbor synaptic distances were in the range of $200 \mathrm{~nm}$. Clearly, this situation is difficult to achieve even with very small single-site terminals.

Closely spaced synapses create a favorable condition for transmitter spillover. Spillover among synapses results in larger charge transfer because of the slower decay of the synaptic currents and reduces the variability of the synaptic transmission (DiGregorio et al., 2002). In the case of synapses separated by 600-700 nm, glutamate spillover still contributes to $50 \%$ of the AMPAmediated synaptic charge in the cerebellum (DiGregorio et al., 2002). In the present study, $80 \%$ of the nearest-neighbor distances were below $300 \mathrm{~nm}$, suggesting a pronounced spillover component. During development, the synapses of the mossy fiber-granule cell contact become more separated, which causes a reduced spillover component of the EPSCs (Cathala et al., 2005), indicating that the exact spatial arrangement of the synapses have strong influence on the extent of spillover.

Cross talk among synapses limits vesicle depletion-based depression at the GABAergic cortico-nuclear synapses in the cerebellum during prolonged high-frequency stimulation (Telgkamp et al., 2004). Interestingly, this effect was mediated by multisite terminals of the Purkinje cells with a mean number of synapses (9.2) similar to those of the rat and monkey SNR terminals (8.5 in 

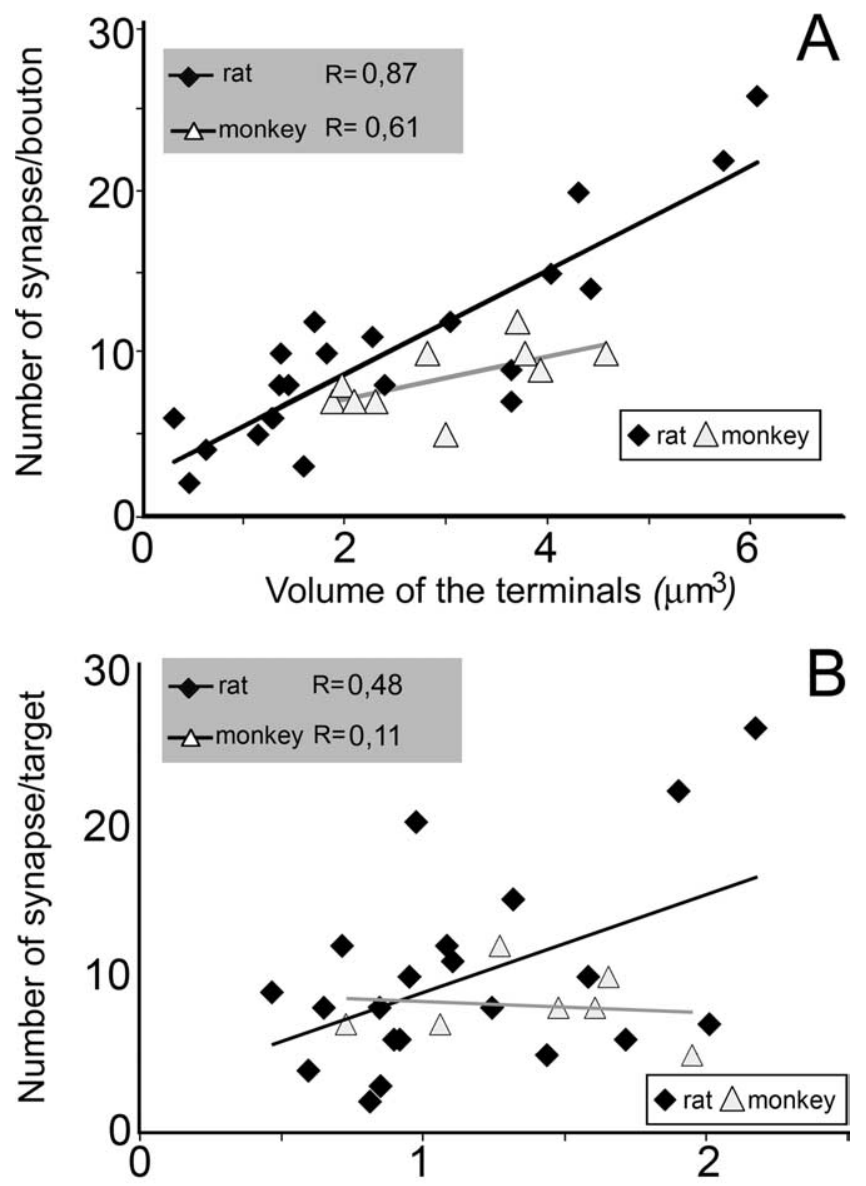

\section{Diameter of the postsynaptic element $(\mu \mathrm{m})$}

Figure 8. Correlation between the number of synapses, the size of the terminals, and the diameter of the postsynaptic target in the two species. $A$, Correlation between the volume of the terminals and the number of synapses per bouton. $\boldsymbol{B}$, Correlation between the diameter of the postsynaptic elements (excluding somatic targets) and the number of synapses with a given target. Rat data, Black diamonds; monkey data, gray triangles. Lines represent the linear regression for each dataset. The data points are in the same range in both species, but only the bouton size and the number of synapses show correlation in case of rats.

this study). Moreover, the average nearest-neighbor distances $(210 \mathrm{~nm})$ were also comparable to our results (rat, $196 \mathrm{~nm}$; monkey, $226 \mathrm{~nm}$ ). Modeling data of Telgkamp et al. (2004) suggested that receptors up to $0.75 \mu \mathrm{m}$ from the release site can bind GABA within $1 \mathrm{~ms}$; thus, the spillover current can contribute not only to the decay but also to the peak of the IPSC. In our study, an average of 3.8 (rat) and 3.4 (monkey) synapses are available from any synapse within $0.75 \mu \mathrm{m}$, making this scenario plausible. Intersynaptic spillover among synapses of the same bouton is able to maintain faithful synaptic transmission at high-frequency firing rates (Telgkamp et al., 2004), when vesicle fusion exceeds the rate of replenishment (Zucker and Regehr, 2002). Similar function can be attributed to the multisite SNR terminals as well. Indeed, the available data on the activity of SNR neurons supports high baseline firing $(50-100 \mathrm{~Hz})$ in awake, behaving monkeys (DeLong et al., 1983; Hikosaka and Wurtz, 1983a; Schultz, 1986).

Our data suggest that glial GABA uptake does not limit intersynaptic diffusion of the transmitter because astrocytic processes did not separate the vast majority of synapses. The nonapposing surface of nigrothalamic boutons, however, was completely ensheated by glia, which certainly limits diffusion among the terminals.
Efficacy of the nigrothalamic pathway

Thalamus is the most important relay station of the basal ganglia output to the neocortex. SNR stimulation induces short-latency, fast IPSPs in the thalamus (Chevalier and Deniau, 1982; Ueki, 1983), consistent with its GABAergic nature. However, because of the strong T-type Ca-channel-mediated rebound response of thalamocortical cells (Jahnsen and Llinas, 1984), SNR activity does not merely induce reduction in the firing rate but promotes burst firing and synchronous discharge, especially in pathophysiological conditions such as Parkinson's disease or epilepsy (Pessiglione et al., 2005; Paz et al., 2007). Indeed, recent in vivo data demonstrated that a decrease in the nigrothalamic output converted rhythmic thalamic burst firing into sustained arrhythmic firing pattern during epileptic spike and wave discharges, showing that GABAergic SNR input to the thalamus participates in the synchronization of thalamocortical cells during seizures (Paz et al., 2007). Interestingly, arrhythmic firing of relay cells disrupted the highly synchronous cortical spike and wave discharges indicating powerful nigral control of cortical activity via the thalamus.

In animal models of Parkinson's disease, the SNR cells display increased burst activity. In addition, a larger proportion of cells participates in oscillatory activity both in rodents and primates (Wichmann et al., 1999; Belluscio et al., 2003). In the basal ganglia, recipient thalamus increased correlation between the activity of thalamic units (Pessiglione et al., 2005), and rhythmic burst discharges during tremor (Guehl et al., 2003) were observed. These data indicate that multisite nigrothalamic terminals described in this study exert strong control on the timing of relay cell firing, which may lead to aberrant cortical activity in pathological conditions.

\section{Are nigrothalamic terminals unique in the forebrain?}

Recent tract-tracing studies disclosed giant multisite GABAergic terminals in the thalamus arising from the zona incerta (Bartho et al., 2002) and anterior pretectal nucleus (APT) (Bokor et al., 2005). Preliminary 3D electron microscopic reconstruction data suggest that the structure of APT terminals is identical to SNR boutons (A. L. Bodor and L. Acsády, unpublished observation). Physiological data demonstrated that similar to SNR boutons, these large GABAergic terminals have powerful influence on the activity of their postsynaptic partner. Stimulation of a single APT fiber in vitro is able to induce rebound bursts in relay cells (Bokor et al., 2005), whereas zona incerta is able to block peripheral information transfer in $\mathrm{n}$. posterior in vivo (Trageser and Keller, 2004; Lavallee et al., 2005). In addition, both zona incerta and APT cells were found to display high-frequency firing in vivo (Bokor et al., 2005; Bartho et al., 2007), emphasizing the link between multisite terminals and fast spiking activity.

\section{Coding in the basal ganglia-thalamus circuit}

Little is known about the behavioral correlates of SNR neuronal activity. Available evidence suggests that neurons not only respond to simple sensory or motor events but also show selective responses when these events are contingent on the behavioral situation or on short-term memory (DeLong et al., 1983; Hikosaka and Wurtz, 1983a,b,c; Schultz, 1986; Wichmann and Kliem, 2004). The response many times can be measured as a subtle change in the firing activity of SNR cells. It may include a decrease or a short pause in the ongoing high-frequency activity, but increase in the firing rate has also been reported (Schultz, 1986; Wichmann and Kliem, 2004). Furthermore, there is uniquely little correlation in spike timing among SNR neurons participat- 
A

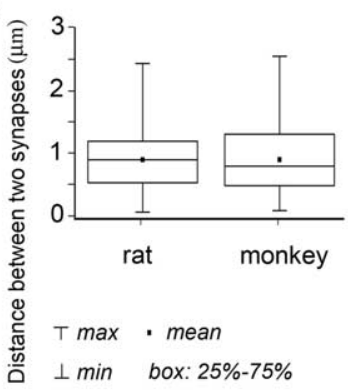

C

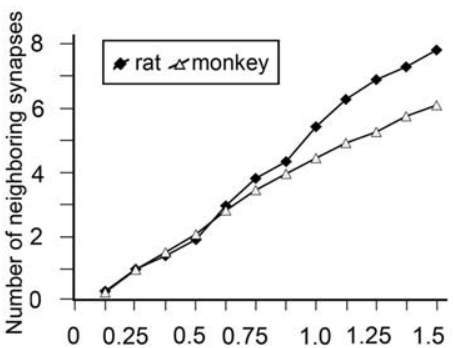

Distance from a given synapse $(\mu \mathrm{m})$
B

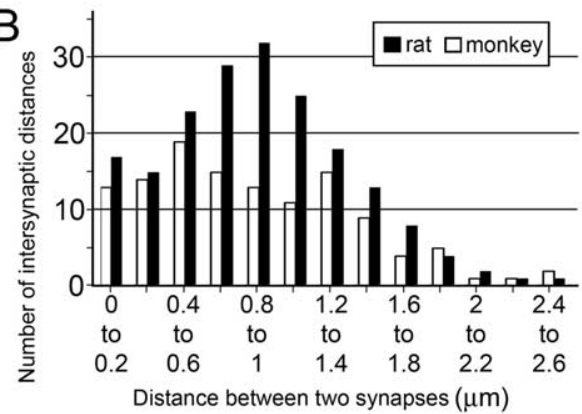

D

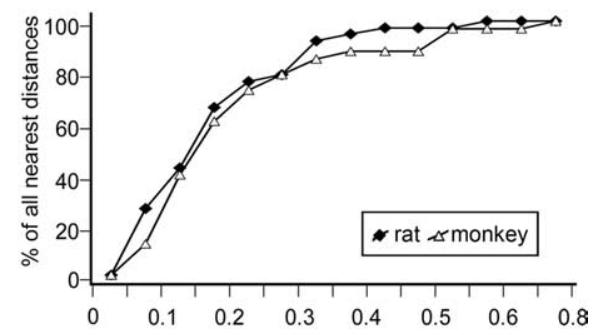

Distance of the nearest neighboring synapse $(\mu \mathrm{m})$

Cerebellar projections compared with those of the ascending somatic sensory pathways in lateral diencephalon. J Comp Neurol 220:229-251.

Berod A, Hartman BK, Pujol JF (1981) Importance of fixation in immunohistochemistry: use of formaldehyde solutions at variable $\mathrm{pH}$ for the localization of tyrosine hydroxylase. J Histochem Cytochem 29:844-850.

Biro AA, Holderith NB, Nusser Z (2006) Release probability-dependent scaling of the postsynaptic responses at single hippocampal GABAergic synapses. J Neurosci 26:12487-12496.

Bokor H, Frere SG, Eyre MD, Slézia A, Ulbert I, Luthi A, Acsády L (2005) Selective GABAergic control of higher-order thalamic relays. Neuron 45:929-940.

Cathala L, Holderith NB, Nusser Z, DiGregorio DA, Cull-Candy SG (2005) Changes in synaptic structure underlie the developmental speeding of AMPA receptor-mediated EPSCs. Nat Neurosci 8:1310-1318.

Chevalier G, Deniau JM (1982) Inhibitory nigral influence on cerebellar evoked responses in the rat ventromedial thalamic nucleus. Exp Brain Res 48:369-376.

Chicurel ME, Harris KM (1992) Threedimensional analysis of the structure and composition of CA3 branched dendritic spines and their synaptic relationships with mossy fiber boutons in the rat hippocampus. J Comp Neurol 325:169-182.

Figure 9. Analysis of the intersynaptic distances in rat and monkey. $A$, Whisker plot of all intersynaptic distances of four reconstructed monkey and rat terminals. $\boldsymbol{B}$, Bar graph showing the same data set as $\boldsymbol{A}$ using $0.2 \mu \mathrm{m}$ bin width. $\boldsymbol{C}$, The average number of neighboring synapses with increasing distances from any given synapse. $\boldsymbol{D}$, Cumulative plot of the nearest-neighbor synaptic distances as the percentage of the total number of nearest-neighbor synaptic distances. Note the closely matched datasets of the two species in $\mathbf{C}$ and $\boldsymbol{D}$.

ing in information transfer (Nevet et al., 2007). These data suggest that postsynaptic thalamic cells should be specifically sensitive to the subtle alteration of the incoming high-frequency GABAergic signal. Our structural data may provide the morphological background for this role. Nigrothalamic terminals preferentially target proximal dendrites, and all of them establish multiple synapses without exception. This may ensure strong and reliable GABAergic transmission and the detection of slight changes in afferent inhibitory input pattern, which may form the structural basis of specific information transfer via inhibition (Person and Perkel, 2005).

\section{References}

Asanuma C, Thach WT, Jones EG (1983) Distribution of cerebellar terminations and their relation to other afferent terminations in the ventral lateral thalamic region of the monkey. Brain Res 286:237-265.

Baimbridge KG, Miller JJ (1982) Immunohistochemical localization of calcium-binding protein in the cerebellum, hippocampal formation and olfactory bulb of the rat. Brain Res 245:223-229.

Bar-Gad I, Morris G, Bergman H (2003) Information processing, dimensionality reduction and reinforcement learning in the basal ganglia. Prog Neurobiol 71:439-473.

Bartho P, Freund TF, Acsády L (2002) Selective GABAergic innervation of thalamic nuclei from zona incerta. Eur J Neurosci 16:999-1014.

Bartho P, Slézia A, Varga V, Bokor H, Pinault D, Buzsaki G, Acsády L (2007) Cortical control of zona incerta. J Neurosci 27:1670-1681.

Beckstead RM, Domesick VB, Nauta WJ (1979) Efferent connections of the substantia nigra and ventral tegmental area in the rat. Brain Res 175:191-217.

Belluscio MA, Kasanetz F, Riquelme LA, Gustavo Murer M (2003) Spreading of slow cortical rhythms to the basal ganglia output nuclei in rats with nigrostriatal lesions. Eur J Neurosci 17:1046-1052.

Berkley KJ (1983) Spatial relationships between the terminations of somatic sensory motor pathways in the rostral brainstem of cats and monkeys. II.
DeLong MR, Crutcher MD, Georgopoulos AP (1983) Relations between movement and single cell discharge in the substantia nigra of the behaving monkey. J Neurosci 3:1599-1606.

Deniau JM, Kita H, Kitai ST (1992) Patterns of termination of cerebellar and basal ganglia efferents in the rat thalamus. Strictly segregated and partly overlapping projections. Neurosci Lett 144:202-206.

Deschenes M, Timofeeva E, Lavallee P (2003) The relay of high-frequency sensory signals in the whisker-to-barreloid pathway. J Neurosci 23:6778-6787.

Di Chiara G, Porceddu ML, Morelli M, Mulas ML, Gessa GL (1979) Evidence for a GABAergic projection from the substantia nigra to the ventromedial thalamus and to the superior colliculus of the rat. Brain Res 176:273-284.

DiGregorio DA, Nusser Z, Silver RA (2002) Spillover of glutamate onto synaptic AMPA receptors enhances fast transmission at a cerebellar synapse. Neuron 35:521-533.

Fremeau Jr RT, Troyer MD, Pahner I, Nygaard GO, Tran CH, Reimer RJ, Bellocchio EE, Fortin D, Storm-Mathisen J, Edwards RH (2001) The expression of vesicular glutamate transporters defines two classes of excitatory synapse. Neuron 31:247-260.

Guehl D, Pessiglione M, Francois C, Yelnik J, Hirsch EC, Feger J, Tremblay L (2003) Tremor-related activity of neurons in the "motor" thalamus: changes in firing rate and pattern in the MPTP vervet model of parkinsonism. Eur J Neurosci 17:2388-2400.

Henze DA, Wittner L, Buzsaki G (2002) Single granule cells reliably discharge targets in the hippocampal CA3 network in vivo. Nat Neurosci 5:790-795.

Hikosaka O, Wurtz RH (1983a) Visual and oculomotor functions of monkey substantia nigra pars reticulata. I. Relation of visual and auditory responses to saccades. J Neurophysiol 49:1230-1253.

Hikosaka O, Wurtz RH (1983b) Visual and oculomotor functions of monkey substantia nigra pars reticulata. II. Visual responses related to fixation of gaze. J Neurophysiol 49:1254-1267.

Hikosaka O, Wurtz RH (1983c) Visual and oculomotor functions of monkey substantia nigra pars reticulata. III. Memory-contingent visual and saccade responses. J Neurophysiol 49:1268-1284.

Hodgson AJ, Penke B, Erdei A, Chubb IW, Somogyi P (1985) Antisera to 
gamma-aminobutyric acid. I. Production and characterization using a new model system. J Histochem Cytochem 33:229-239.

Ilinsky IA, Kultas-Ilinsky K (1987) Sagittal cytoarchitectonic maps of the Macaca mulatta thalamus with a revised nomenclature of the motorrelated nuclei validated by observations on their connectivity. J Comp Neurol 262:331-364.

Ilinsky IA, Kultas-Ilinsky K (1990) Fine structure of the magnocellular subdivision of the ventral anterior thalamic nucleus (VAmc) of Macaca mulatta: I. Cell types and synaptology. J Comp Neurol 294:455-478.

Ilinsky IA, Jouandet ML, Goldman-Rakic PS (1985) Organization of the nigrothalamocortical system in the rhesus monkey. J Comp Neurol 236:315-330.

Ilinsky IA, Yi H, Kultas-Ilinsky K (1997) Mode of termination of pallidal afferents to the thalamus: a light and electron microscopic study with anterograde tracers and immunocytochemistry in Macaca mulatta. J Comp Neurol 386:601-612.

Ilinsky IA, Ambardekar AV, Kultas-Ilinsky K (1999) Organization of projections from the anterior pole of the nucleus reticularis thalami (NRT) to subdivisions of the motor thalamus: light and electron microscopic studies in the rhesus monkey. J Comp Neurol 409:369-384.

Jahnsen H, Llinas R (1984) Electrophysiological properties of guinea-pig thalamic neurones: an in vitro study. J Physiol (Lond) 349:205-226.

Kalil K (1981) Projections of the cerebellar and dorsal column nuclei upon the thalamus of the rhesus monkey. J Comp Neurol 195:25-50.

Kubota Y, Kawaguchi Y (2000) Dependence of GABAergic synaptic areas on the interneuron type and target size. J Neurosci 20:375-386.

Kultas-Ilinsky K, Ilinsky IA (1990) Fine structure of the magnocellular subdivision of the ventral anterior thalamic nucleus (VAmc) of Macaca mulatta: II. Organization of nigrothalamic afferents as revealed with EM autoradiography. J Comp Neurol 294:479-489.

Kuroda M, Price JL (1991) Ultrastructure and synaptic organization of axon terminals from brainstem structures to the mediodorsal thalamic nucleus of the rat. J Comp Neurol 313:539-552.

Land PW, Kyonka E, Shamalla-Hannah L (2004) Vesicular glutamate transporters in the lateral geniculate nucleus: expression of VGLUT2 by retinal terminals. Brain Res 996:251-254.

Lavallee P, Urbain N, Dufresne C, Bokor H, Acsády L, Deschenes M (2005) Feedforward inhibitory control of sensory information in higher-order thalamic nuclei. J Neurosci 25:7489-7498.

Lieberman AR, Spacek J (1997) Filamentous contacts: the ultrastructure and three-dimensional organization of specialized non-synaptic interneuronal appositions in thalamic relay nuclei. Cell Tissue Res 288:43-57.

Nevet A, Morris G, Saban G, Arkadir D, Bergman H (2007) Lack of spikecount and spike-time correlations in the substantia nigra reticulata despite overlap of neural responses. J Neurophysiol 98:2232-2243.

Ohara PT, Lieberman AR (1993) Some aspects of the synaptic circuitry underlying inhibition in the ventrobasal thalamus. J Neurocytol 22:815-825.

Paxinos G, Watson C (1998) The rat brain in stereotaxic coordinates, Ed 4. London: Academic.

Paxinos G, Huang XF, Toga AW (2000) The rhesus monkey brain in stereotaxic coordinates. London: Academic.

Paz JT, Chavez M, Saillet S, Deniau JM, Charpier S (2007) Activity of ventral medial thalamic neurons during absence seizures and modulation of cortical paroxysms by the nigrothalamic pathway. J Neurosci 27:929-941.

Percheron G, Francois C, Talbi B, Yelnik J, Fenelon G (1996) The primate motor thalamus. Brain Res Rev 22:93-181.

Person AL, Perkel DJ (2005) Unitary IPSPs drive precise thalamic spiking in a circuit required for learning. Neuron 46:129-140.

Pessiglione M, Guehl D, Rolland AS, Francois C, Hirsch EC, Feger J, Tremblay L (2005) Thalamic neuronal activity in dopamine-depleted primates: evidence for a loss of functional segregation within basal ganglia circuits. J Neurosci 25:1523-1531.
Rollenhagen A, Lübke JH (2006) The morphology of excitatory central synapses: from structure to function. Cell Tissue Res 326:221-237.

Rouiller EM, Liang F, Babalian A, Moret V, Wiesendanger M (1994) Cerebellothalamocortical and pallidothalamocortical projections to the primary and supplementary motor cortical areas: a multiple tracing study in macaque monkeys. J Comp Neurol 345:185-213.

Sakai ST, Inase M, Tanji J (1996) Comparison of cerebellothalamic and pallidothalamic projections in the monkey (Macaca fuscata): a double anterograde labeling study. J Comp Neurol 368:215-228.

Sakai ST, Grofova I, Bruce K (1998) Nigrothalamic projections and nigrothalamocortical pathway to the medial agranular cortex in the rat: singleand double-labeling light and electron microscopic studies. J Comp Neurol 391:506-525.

Satzler K, Sohl LF, Bollmann JH, Borst JG, Frotscher M, Sakmann B, Lübke JH (2002) Three-dimensional reconstruction of a calyx of Held and its postsynaptic principal neuron in the medial nucleus of the trapezoid body. J Neurosci 22:10567-10579.

Schultz W (1986) Activity of pars reticulata neurons of monkey substantia nigra in relation to motor, sensory, and complex events. J Neurophysiol 55:660-677.

Shepherd GM, Harris KM (1998) Three-dimensional structure and composition of CA3-CA1 axons in rat hippocampal slices: implications for presynaptic connectivity and compartmentalization. J Neurosci 18:8300-8310.

Sherman SM, Guillery RW (1998) On the actions that one nerve cell can have on another: distinguishing "drivers" from "modulators." Proc Natl Acad Sci USA 95:7121-7126.

Smith PH, Joris PX, Yin TC (1998) Anatomy and physiology of principal cells of the medial nucleus of the trapezoid body (MNTB) of the cat. J Neurophysiol 79:3127-3142.

Somogyi P, Hodgson AJ, Chubb IW, Penke B, Erdei A (1985) Antisera to gamma-aminobutyric acid. II. Immunocytochemical application to the central nervous system. J Histochem Cytochem 33:240-248.

Taschenberger H, Leao RM, Rowland KC, Spirou GA, von Gersdorff H (2002) Optimizing synaptic architecture and efficiency for highfrequency transmission. Neuron 36:1127-1143.

Telgkamp P, Padgett DE, Ledoux VA, Woolley CS, Raman IM (2004) Maintenance of high-frequency transmission at purkinje to cerebellar nuclear synapses by spillover from boutons with multiple release sites. Neuron 41:113-126.

Tepper JM, Abercrombie ED, Bolam JP (2007) Basal ganglia macrocircuits. Prog Brain Res 160:3-7.

Tracey DJ, Asanuma C, Jones EG, Porter R (1980) Thalamic relay to motor cortex: afferent pathways from brain stem, cerebellum, and spinal cord in monkeys. J Neurophysiol 44:532-554.

Trageser JC, Keller A (2004) Reducing the uncertainty: gating of peripheral inputs by zona incerta. J Neurosci 24:8911-8915.

Tsumori T, Yokota S, Ono K, Yasui Y (2002) Synaptic organization of GABAergic projections from the substantia nigra pars reticulata and the reticular thalamic nucleus to the parafascicular thalamic nucleus in the rat. Brain Res 957:231-241.

Ueki A (1983) The mode of nigro-thalamic transmission investigated with intracellular recording in the cat. Exp Brain Res 49:116-124.

Wichmann T, Kliem MA (2004) Neuronal activity in the primate substantia nigra pars reticulata during the performance of simple and memoryguided elbow movements. J Neurophysiol 91:815-827.

Wichmann T, Bergman H, Starr PA, Subramanian T, Watts RL, DeLong MR (1999) Comparison of MPTP-induced changes in spontaneous neuronal discharge in the internal pallidal segment and in the substantia nigra pars reticulata in primates. Exp Brain Res 125:397-409.

Zucker RS, Regehr WG (2002) Short-term synaptic plasticity. Annu Rev Physiol 64:355-405. 